\title{
Comparison of Electronic Structure and Magnetic Properties of Few Layer Graphene and Multiwall Carbon Nanotubes
}

\author{
Sekhar Chandra Ray \\ Department of Physics, College of Science, Engineering and Technology, University of South Africa, Science Campus, \\ Private Bag X6, Christiaan de Wet and Pioneer Avenue, Florida Park, Florida, Johannesburg 1710, South Africa
}

Correspondence should be addressed to Sekhar Chandra Ray; raysc@unisa.ac.za

Received 5 July 2016; Accepted 5 October 2016

Academic Editor: Luigi Nicolais

Copyright (c) 2016 Sekhar Chandra Ray. This is an open access article distributed under the Creative Commons Attribution License, which permits unrestricted use, distribution, and reproduction in any medium, provided the original work is properly cited.

A comparative study has been made for the non-catalyst based few layer graphene (FLG) and Fe-catalyst based multiwall carbon nanotubes (MWCNTs). Magnetic and electronic properties of FLG and MWCNTs were studied using magnetic M-H hysteresis loops and synchrotron radiation based X-ray absorption fine structure spectroscopy measurements. Structural defects and electronic and bonding properties of FLG/MWCNTs have been studied using Raman spectroscopy, X-ray photoelectron spectroscopy (XPS), and ultraviolet photoelectron spectroscopy (UPS). The work functions of FLG and MWCNTs are $4.01 \mathrm{eV}$ and $3.79 \mathrm{eV}$, respectively, obtained from UPS (He-I) spectra. UPS (He-II) results suggest that the density of states (DOS) of MWCNTs is higher than FLG and is consistent with Raman spectroscopy result that shows the defect of MWCNTs is higher than FLG. The magnetic coercivity $\left(H_{c}\right)$ of the MWCNTs $(\sim 750 \mathrm{Oe})$ is higher than FLG $(\sim 85 \mathrm{Oe})$ which could be used for various technological magnetic applications.

\section{Introduction}

Graphene, a one-atom thick sheet of carbon atoms arranged in a hexagonal lattice, has many desirable properties. It has become vey useful for the electronic and magnetic-storage devices [1-7]. Graphene materials have long spin relaxation time and length due to the small spin-orbit coupling, so graphene is most likely used for the spintronics applications. Spintronics is a field of research, where both the fundamental research and innovative device applications coexist with each other. Spintronics devices are pervasive, and most notably they can be found in computers' hard disks. They function due to coupling of magnetism and electric current $[6,7]$. Similar to the modulation of physical and chemical properties of carbon nanotubes, graphene has been proposed for versatile applications [8-10]. In the other way, the ferromagnetic materials such as "Fe-"catalyst based carbon nanotubes (CNTs) have potential application in various areas such as "magneticstorage media" that include the traditional tapes and videocassettes, hard disks for mainframe computers, floppy disks for personal computers (PC), and portable ZIP and MO disks (magnetooptic) with high storage capacity [11, 12]. So it is important to study the magnetic properties of carbon nanostructured materials like graphene/few layer graphene (FLG) and carbon nanotubes (CNTs)/multiwall-CNTs for these applications.

In this work, we have studied the few layer graphene (FLG) and multiwall carbon nanotubes (MWCNTs) to elucidate their electronic structure, bonding properties, and magnetic behaviours and compared them with a view on the magnetic device application like low-dimensional high-density magnetic recording media.

\section{Experimental Details}

Microwave plasma enhanced chemical vapor deposition (PECVD) process was used to grow the non-catalyst based vertically aligned few layers graphene (FLG) thin films on bare $\mathrm{Si}$ (100) substrates using $\mathrm{CH}_{4}$ as carbon precursor, whereas the MWCNTs were grown by catalytical chemical vapor deposition (CVD) using camphor (as carbon source) and the ferrocene (Fe-catalyst) in the ratio of $20: 1$. Details 
of growing process of FLG and MWCNTs could be available elsewhere $[13,14]$. The surface morphology was measured using scanning electron microscopy. The electronic properties were measured using X-ray absorption near edge structure spectroscopy (XANES), X-ray photoelectron spectroscopy (XPS), and ultraviolet photoelectron spectroscopy (UPS). The microstructure and defects are studied using Raman spectroscopy and electron field emission, whereas the field-dependent magnetization of FLG and MWCNTs was studied using a SQUID-type magnetometer. The core-level C 1s X-ray photoelectron microscopy (XPS) and ultraviolet photoemission spectroscopy (UPS) measurements of the FLG and MWCNT were performed on the monochromatic Al $K_{\alpha}=1486.6 \mathrm{eV}$ excitation energy and a He lamp (HeII) with $40.8 \mathrm{eV}$ excitation energy, respectively, using the KRATOS-AXIS SUPRA X-ray photoelectron spectroscopy (XPS) equipment at UNISA (Florida Science Campus), South Africa. Work functions were determined from the UPS HeI spectra using excitation energy $21.22 \mathrm{eV}$. Raman spectra measurements were carried out using $647 \mathrm{~nm}$ excitation wavelengths with the LASER beam spot size $\sim 1 \mu \mathrm{m}$ and the incident power was $\sim 1 \mathrm{~mW}$.

\section{Results and Discussion}

The scanning electron microscopy (SEM) images of FLG and MWCNTs are shown in Figures 1(a) and 1(b). It is evident that the synthesized graphene is vertically aligned to the underlying substrate and is randomly intercalated forming a porous mesh-like network. In case of MWCNTs, most of the nanotubes are not completely vertically aligned and some of them are entangled. Figure 1(b) reveals that the Fe nanoparticles are embedded in the end of the MWCNTs, offering excellent oxidation protection, which can be effectively exploited in magnetic nanoprobes and spintronics application. Figures 1(c) and 1(d) display the electron field emission (EFE) measurements of emitted current density $(J)$ and applied electric field $\left(E_{A}\right)$ and inset shows the corresponding FowlerNordheim (F-N) plots of FLG and MWCNTs, respectively. The figure shows that there exists a threshold of electric field, beyond which $J$ increases roughly exponentially. FowlerNordheim (F-N) plots (see inset figure) clearly illustrate the threshold electric field or turn-on electric field $\left(E_{\mathrm{TOE}}\right)$. The values of $E_{\mathrm{TOE}}$ were obtained with linear curve fitting in the high electric field region and were found to be $26.5 \mathrm{~V} / \mu \mathrm{m}$ for FLG and $4.7 \mathrm{~V} / \mu \mathrm{m}$ for MWCNTs indicating that MWCNTs have better field emission properties than FLG. It is noted that $E_{\mathrm{TOE}}$ is defined as the field at which $J$ exceeds $0.1 \mu \mathrm{A} / \mathrm{cm}^{2}$ and is deduced from a linear fitting of the F-N plots in the high applied electric field. The notable feature of MWCNTs shows that the current density $(J)$ is $3.82 \mathrm{~mA} / \mathrm{cm}^{2}$, when the electric field is $10 \mathrm{~V} / \mu \mathrm{m}$, whereas no current is observed in case of FLG at electric field $10 \mathrm{~V} / \mu \mathrm{m}$. X-ray photoemission images of FLG and MWCNT are shown in Figures 1(e) and 1(f), respectively, where red particles are carbon atoms and blue particles are oxygen atoms which are obtained at the kinetic energy of $\sim 1202.00 \mathrm{eV}$ (C 1s) and $954.14 \mathrm{eV}$ (O 1s), respectively. These images show the uniform distribution of carbon and oxygen atoms on the FLG and MWCNTs surface. The images further imply that the oxygen atoms are less in MWCNTs compared to FLG.

Figure 2(a) shows the Raman spectra of pristine FLG graphene and MWCNTs. The Raman spectra of MWCNTs, the most prominent bands of interest, are (i) the D band at $\sim 1360 \mathrm{~cm}^{-1}$, which is disorder-activated with $A_{1 \mathrm{~g}}$ symmetry, (ii) the $\mathrm{G}$ band at $\sim 1580 \mathrm{~cm}^{-1}$, assigned to the Raman-allowed phonon mode with $E_{2 g}$ symmetry, and (iii) the first overtone of the $\mathrm{D}$ band, that is, $2 \mathrm{D}$ band at $\sim 2700 \mathrm{~cm}^{-1}$ [15]. In case of the FLG, the characteristic peaks are $G$ band at $\sim 1583 \mathrm{~cm}^{-1}$ and $2 \mathrm{D}$ band at $\sim 2664 \mathrm{~cm}^{-1}$ [15]. The other defects induced peaks at $\sim 1335$ and $1617 \mathrm{~cm}^{-1}$ are also observed, which have been assigned to $\mathrm{D}$ band and $\mathrm{D}^{\prime}$ band activated by defects via double-resonance process [15]. The $\mathrm{D}$ peak is defect activated via an intervalley double-resonance process and its intensity provides a convenient measure for the amount of disorder [16-20]. The peaks at $2460 \mathrm{~cm}^{-1}$ arise in both FLG and MWCNTs via a combination of $\left(D+D^{\prime}\right)$ bands and are defect activated [21-23]. Commonly, the intensity ratio of $\mathrm{D}$ band over $\mathrm{G}$ band $I_{\mathrm{D}} / I_{\mathrm{G}}$ can serve as a convenient measurement of the amount of defects in graphitic materials [17]. The $I_{\mathrm{D}} / I_{\mathrm{G}}$ ratio of MWCNTs and FLG are 0.70 and 0.44 , respectively, indicating MWCNTs have higher defects than FLG.

Figure 2(b) displays the C K-edge XANES spectra of FLG and MWCNTs and the reference graphite. The $\mathrm{C} K$-edge XANES spectra of reference graphite are vertically shifted for clarity. For graphitic materials in general, X-ray absorption near edge structure (XANES) spectra can be divided into three regions characterized by specific resonance energies [21]. The first region of $\pi^{*}$ resonance appears around $285 \pm$ $1 \mathrm{eV}$ and the $\mathrm{C}-\mathrm{H}^{*}$ resonance appears around $288 \pm 1 \mathrm{eV}$, and a broad region between $290 \mathrm{eV}$ and $315 \mathrm{eV}$ corresponds to $\sigma^{*}$ resonance. The presence of the $\pi^{*}$ and $\mathrm{C}-\mathrm{H}^{*}$ resonances serves as a fingerprint for the existence of $\mathrm{sp}^{2}$ hybridized $\mathrm{C}$ $\mathrm{C}$ bonds and $\mathrm{C}-\mathrm{H}$ bonds, respectively. These spectra reflect a transition from the $\mathrm{C} 1 \mathrm{~s}$ core state to the $p$-like final states above the Fermi level. The $\pi^{*}$ features associated with $s p^{2} \mathrm{C}$ of the graphite; MWCNTs are at $\sim 285.5,286.3$, and $286.1 \mathrm{eV}$, respectively, whereas the $\sigma^{*}$ feature observed at $\sim 293 \mathrm{eV}$ is assigned to the $s p^{3} \mathrm{C}$ states $[21,22]$. The $\mathrm{C} K$-edge XANES spectra of the FLG show features at $\sim 286.1 \mathrm{eV}, \sim 292.6 \mathrm{eV}$, and $\sim 291.6 \mathrm{eV}$ which can be attributed to the unoccupied $1 \mathrm{~s} \rightarrow$ $\pi^{*}, 1 \mathrm{~s} \rightarrow \sigma^{*}$, and excitonic states transitions, respectively $[22,23]$. The peak at 288.1 is defined as $\mathrm{C}-\mathrm{H}$ and the peak at $\sim 288.9 \mathrm{eV}$ is the interlayer graphite structure and these two peaks are not prominent in the MWCNTs.

Figure 3 shows the $\mathrm{C}$ 1s core-level spectra of pristine FLG and MWCNTs. The C 1s spectra of FLG and MWCNTs are fitted with three Gaussian components. In case of FLG, the peak at $\sim 284.4 \mathrm{eV}$ assigned to $\mathrm{sp}^{2}$ hybridized $\mathrm{C}$ atoms in graphene, the peak at $\sim 285.2 \mathrm{eV}$ assigned to $\mathrm{sp}^{3}$ that hybridized $\mathrm{C}$ atoms arises from C-H and C-C bonds, and the peak at $\sim 286.3 \mathrm{eV}$ was assigned as $\mathrm{C}-\mathrm{O}$ bonds. All these peaks are shifted $\sim 0.19 \mathrm{eV}$ for MWCNTs. The work functions determined from He-I spectra $(\sim 40.8 \mathrm{eV})$ as shown in Figure 4 . The work functions are estimated from the linear fitting of the edge of spectra as shown in inset Figure 4. The estimated work functions of FLG and MWCNTs are $4.01 \mathrm{eV}$ and $3.79 \mathrm{eV}$, 


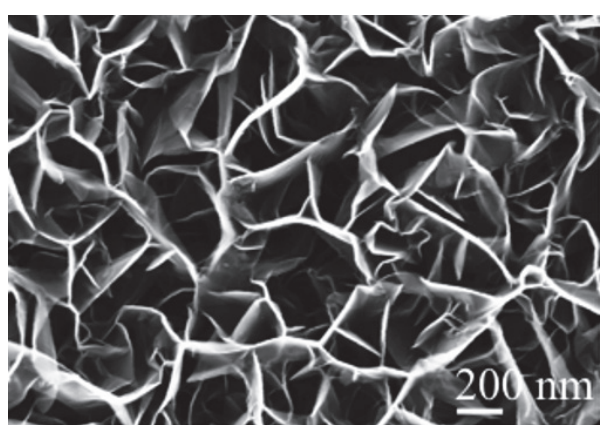

(a)

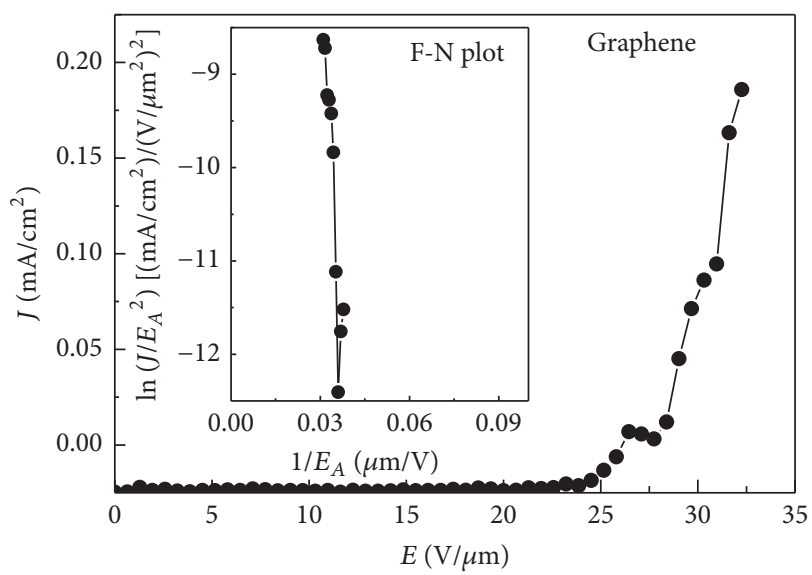

(c)

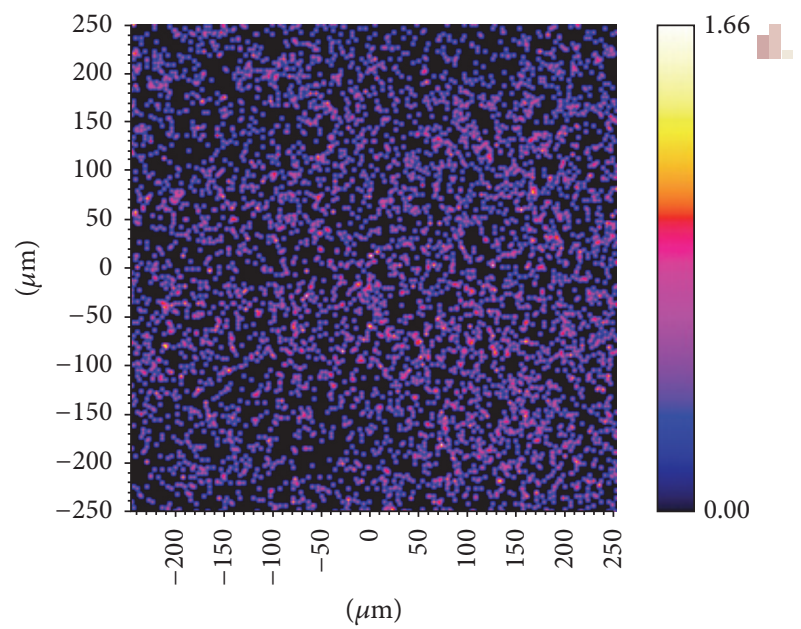

(e)

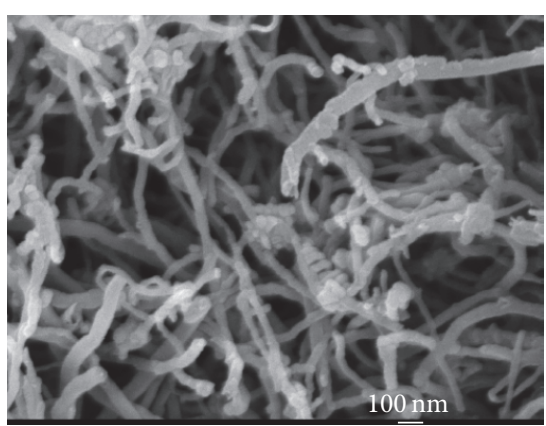

(b)

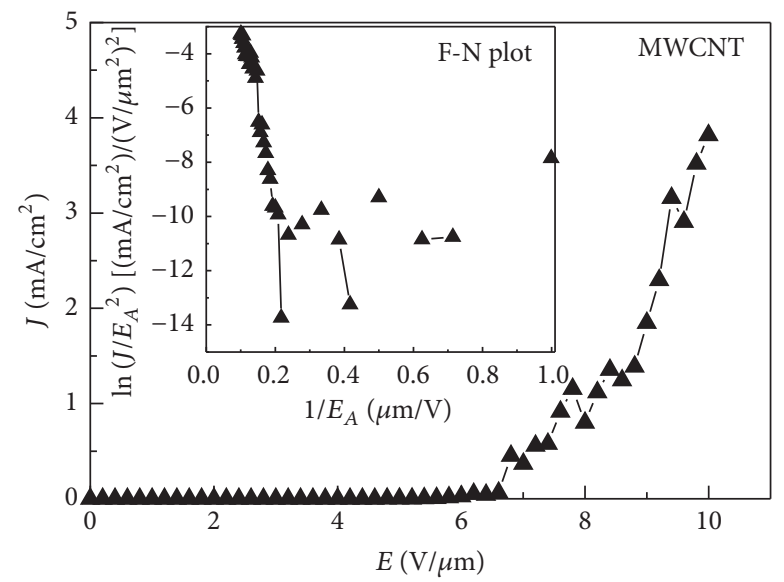

(d)

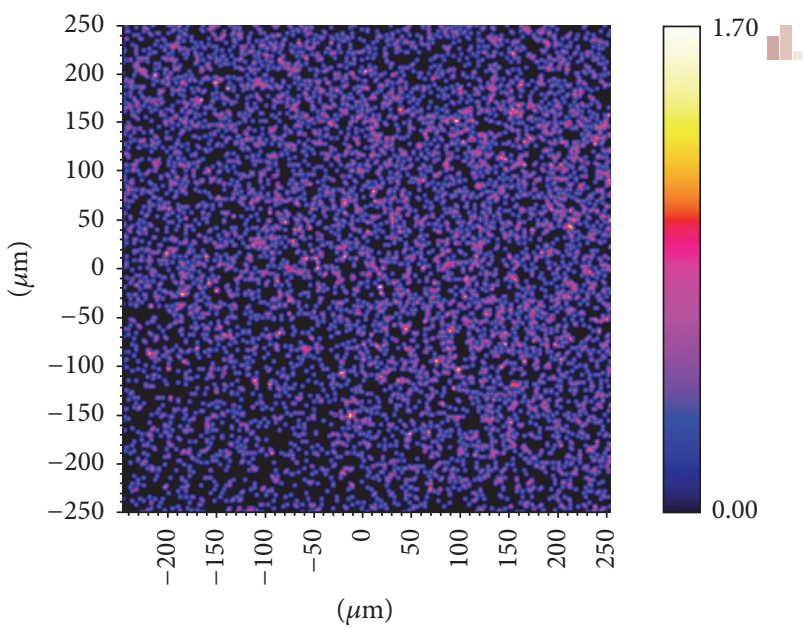

(f)

FIGURE 1: Scanning electron microscopy of (a) FLG, (b) MWCNTs, and electron field emission (EFE) of (c) FLG and (d) MWCNTs. XPS image of (e) graphene and (f) MWCNT [red particles are carbon and blue particles are oxygen in both cases] obtained when kinetic energy is $1202.00 \mathrm{eV}$ (C 1s) and $954.14 \mathrm{eV}$ (O 1s), respectively.

respectively, which are comparable with the values reported by Ago et al. [24]. As shown in Figure 5, the UPS spectra corresponding to valence-band density of states (DOS) of FLG and MWCNTs obtained at He-II radiation $(\sim 40.8 \mathrm{eV})$. These spectra exhibit significant changes in electron states below the Fermi level after being normalized to possess the same integrated intensity with respect to $0-19 \mathrm{eV}$ binding energies. The spectrum of pristine FLG shows five band features, which are assigned to (i) $\mathrm{C} 2 \mathrm{p} \pi$ at $\sim 4.8 \mathrm{eV}$, (ii) crossing of $\mathrm{C} 2 \mathrm{p} \pi$ and $\mathrm{C} 2 \mathrm{p} \sigma$ bands around $6.7 \mathrm{eV}$, (iii) $\mathrm{C}$ $2 \mathrm{p} \sigma$ at $9.2 \mathrm{eV}$, (iv) C $2 \mathrm{~s}-2 \mathrm{p}$ hybridized state at $11.9 \mathrm{eV}$, and (v) $\mathrm{C} 2 \mathrm{~s} \sigma$ band at $\sim 13.5 \mathrm{eV}$ [25]. In case of MWCNTs, all these peaks are shifted $\sim 0.15 \mathrm{eV}$ as indicated that the Fermi edge is shifted $\sim 0.15 \mathrm{eV}$ as shown in inset Figure 3 that further implies 


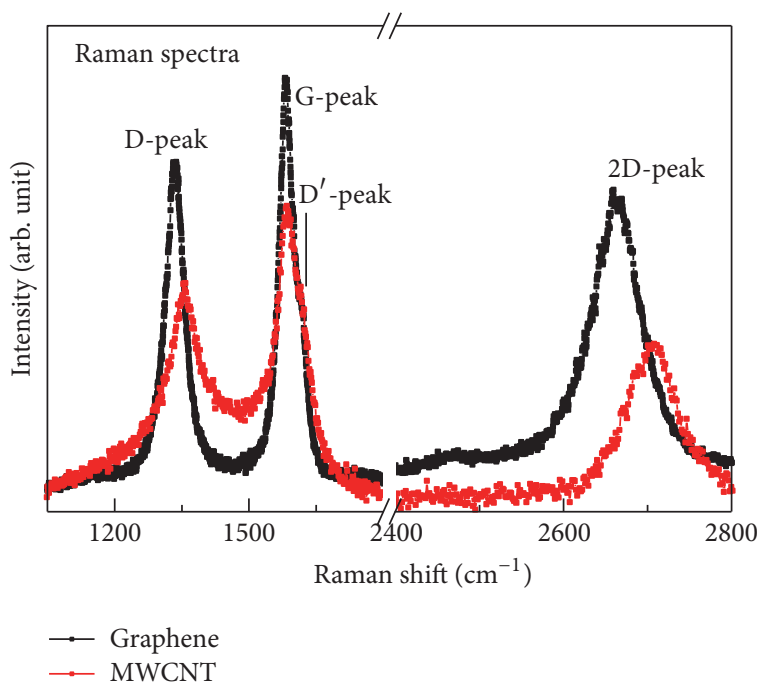

(a)

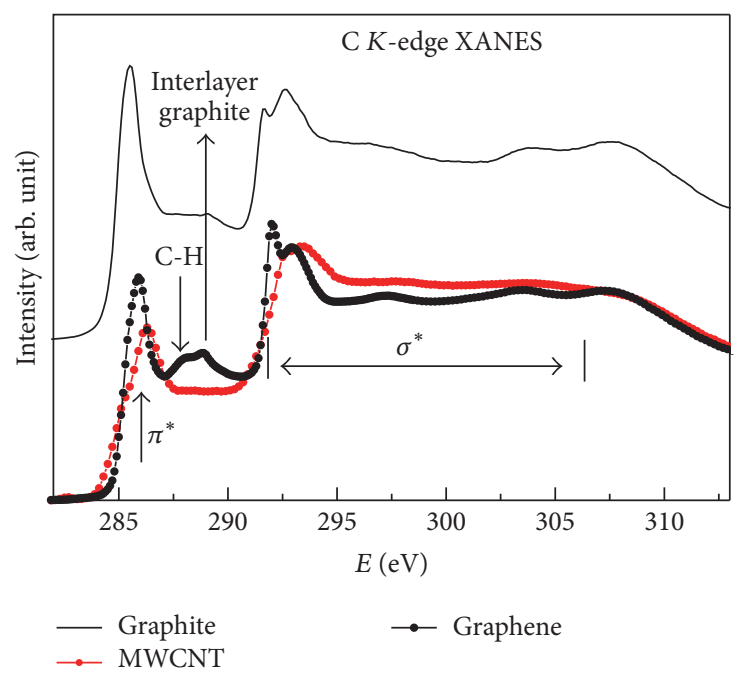

(b)

FIGURE 2: Raman spectroscopy of (a) FLG and MWCNTs and C K-edge XANES spectroscopy of (b) FLG and MWCNTs along with graphite as a reference.
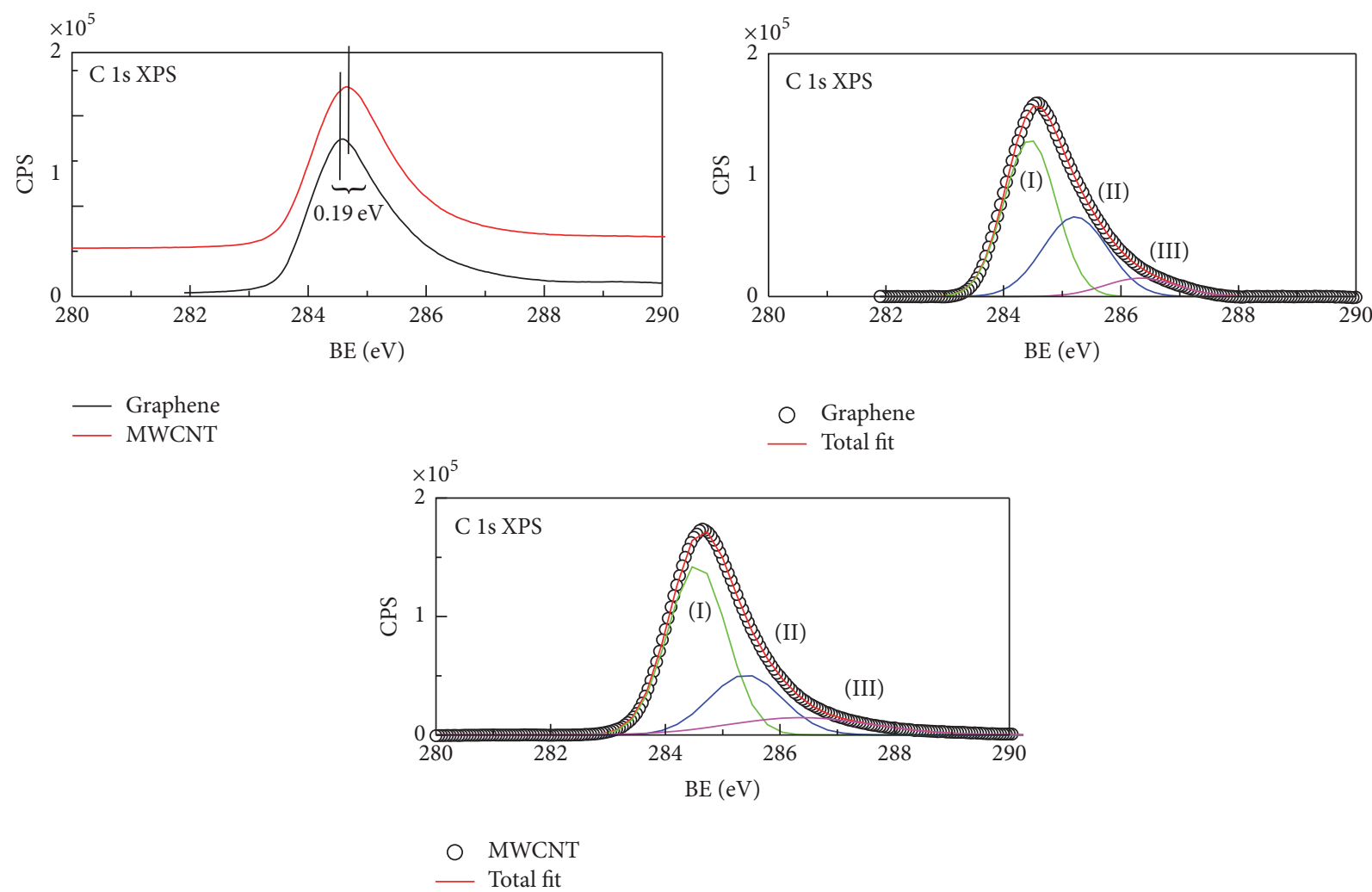

FIGURE 3: C 1s XPS spectra of graphene and MWCNTs. Deconvolution into three Gaussian components for each FLG and MWCNTs.

reducing the work function. It is also observed that the relative intensity of the $\mathrm{p} \pi$ - and $\mathrm{p} \sigma$-derived DOS of MWCNTs is higher than FLG. This could be accounted for by the presence of defects sites, as identified in the Raman spectra.

PPMS were used at room temperature $(300 \mathrm{~K})$, to investigate the hysteresis loop of the magnetic behaviour. Figures 6(a) and 6(b) plot the resulting magnetic moment (M) and $\mu_{0} \mathrm{H}$ of as-grown MWCNTs and FLG, respectively. The magnetic moment of the FLG is nearly $10^{4}$ times lower than those of MWCNTs. However, we strongly believe the higher ferromagnetic behaviour in MWCNTs is attributed due to Fe nanoparticles. The coercivity $\left(H_{c}\right)$ of the MWCNTs is 


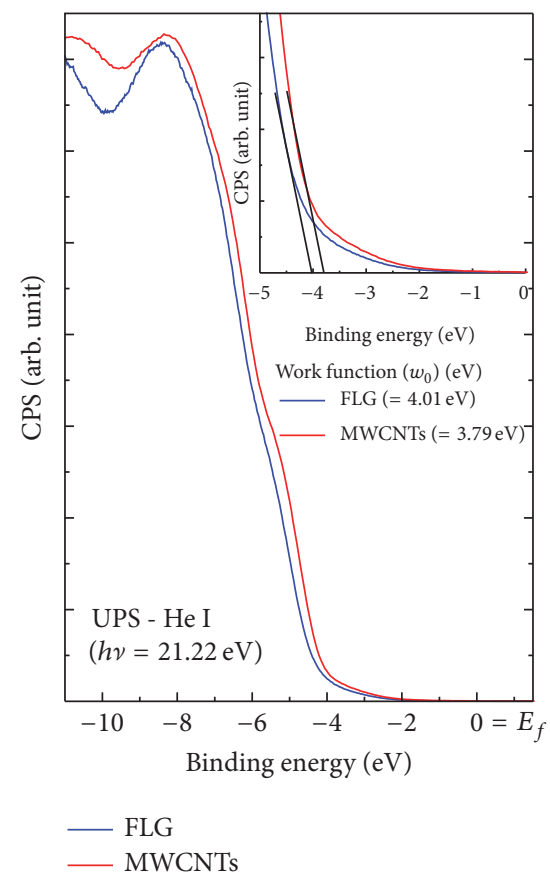

Figure 4: UPS spectra (He I = 21.22 eV) of graphene and MWCNTs. Estimated work functions of FLG and MWCNTs are $4.01 \mathrm{eV}$ and $3.79 \mathrm{eV}$, respectively.
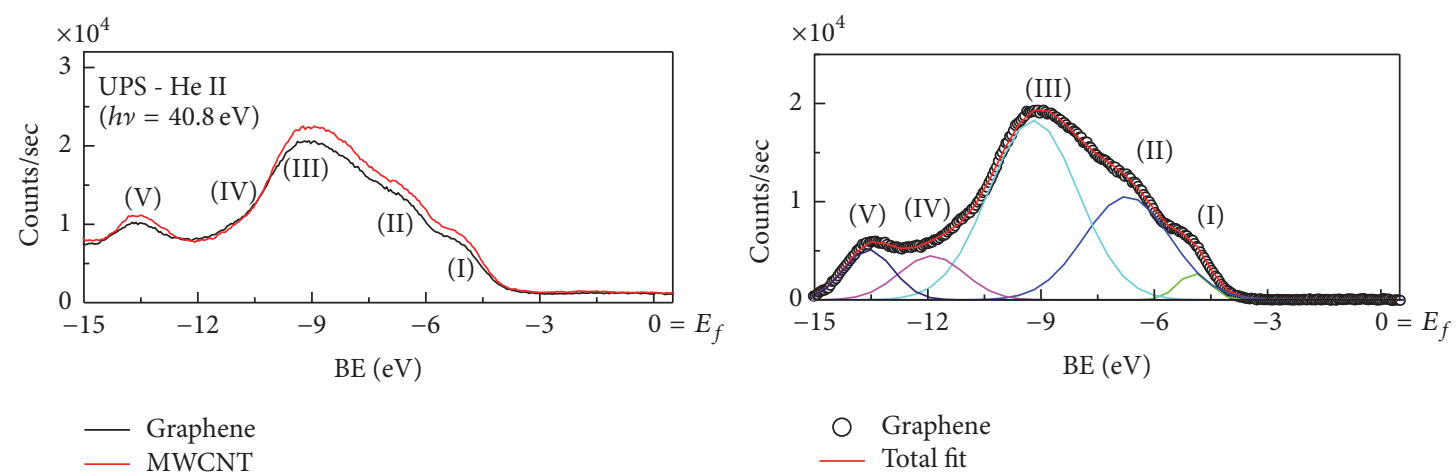

O Graphene

- Total fit

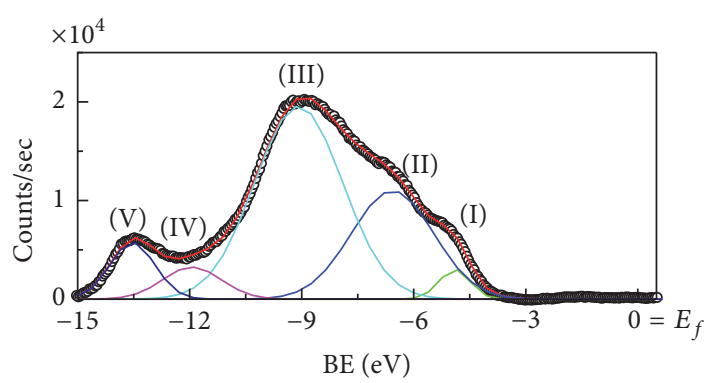

MWCNT

Figure 5: UPS spectra $(\mathrm{He}-\mathrm{II}=40.8 \mathrm{eV})$ of graphene and MWCNTs. Deconvolution into five Gaussian components for each FLG and MWCNTs. 


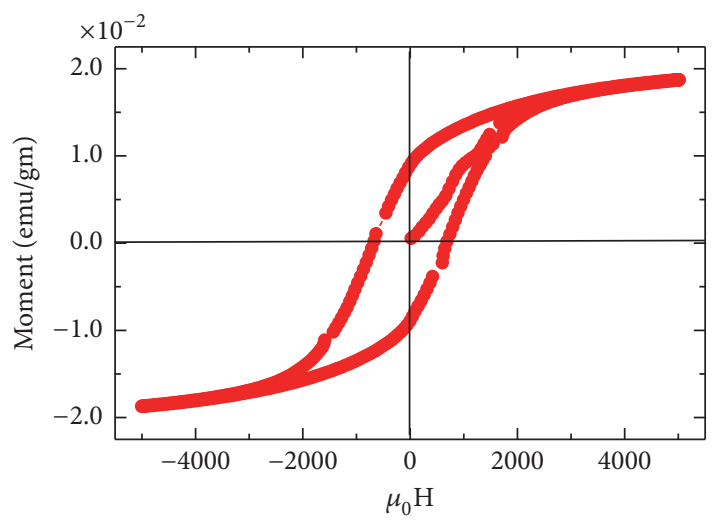

(a) MWCNT

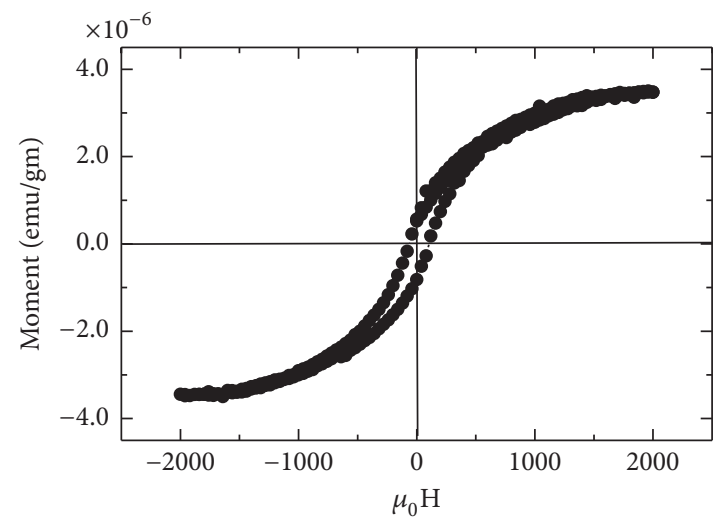

(b) Graphene

FIGURE 6: Field-dependent magnetization M-H hysteresis loop of (a) MWCNTs and (b) FLG.

$\sim 750$ Oe that is counterpart of bulk Fe (0.9 Oe) [26] and Ni and Co nanowire arrays $[23,27]$ and it is comparable with values obtained elsewhere $[28,29]$. On the other hand, the coercivity $\left(H_{c}\right)$ of the FLG is $\sim 85$ Oe and is nine times lower than MWCNT. The difference of these magnetic properties is due to the differences of defects, DOS, and work functions. These findings are encouraging for various technological applications and suggest higher magnetic stability of these graphene-based FLG and MWCNTs materials.

\section{Summary and Conclusion}

In summary, we have studied the electronic and magnetic properties of MWCNTs and FLG. We found that the defects and DOS are higher in MWCNTs than FLG in this study. The work functions of FLG and MWCNTs are $4.01 \mathrm{eV}$ and $3.79 \mathrm{eV}$, respectively. The room temperature magnetization of MWCNTs is found to be $\sim 10^{4}$ times higher than FLG. The coercivity $(\mathrm{Hc})$ of the MWCNTs is also higher than FLG. These ferromagnetic properties could open up the possibility of producing highly tunable graphene-based applications devices, including spintronic nanodevices, magnetoresistance, and magnetic memory devices. The results of MWCNTs also demonstrate the potential applications in magnetic recording media of the isolated and well-distributed magnetic particles in the magnetic metal-encapsulated carbon nanostructures.

\section{Competing Interests}

The authors declare no competing financial interests and no conflict of interests regarding the publication of this paper.

\section{Acknowledgments}

The author Sekhar Chandra Ray acknowledges National Research Foundation, South Africa, for financial support.

\section{References}

[1] S. C. Ray, N. Soin, T. Makgato et al., "Graphene supported graphone/graphane bilayer nanostructure material for spintronics," Scientific Reports, vol. 4, article 3862, 2014.

[2] K. S. Novoselov, A. K. Geim, S. V. Morozov et al., "Electric field in atomically thin carbon films," Science, vol. 306, no. 5696, pp. 666-669, 2004.

[3] A. K. Geim, “Graphene: status and prospects," Science, vol. 324, no. 5934, pp. 1530-1534, 2009.

[4] M. H. Gass, U. Bangert, A. L. Bleloch, P. Wang, R. R. Nair, and A. K. Geim, "Free-standing graphene at atomic resolution," Nature Nanotechnology, vol. 3, no. 11, pp. 676-681, 2008.

[5] S. Stankovich, D. A. Dikin, G. H. B. Dommett et al., "Graphenebased composite materials," Nature, vol. 442 , no. 7100 , pp. 282 286, 2006.

[6] L. Li, R. Qin, H. Li et al., "Functionalized graphene for highperformance two-dimensional spintronics devices," ACS Nano, vol. 5, no. 4, pp. 2601-2610, 2011.

[7] A. J. Hong, E. B. Song, H. S. Yu et al., "Graphene flash memory," ACS Nano, vol. 5, no. 10, pp. 7812-7817, 2011.

[8] J. O. Sofo, A. S. Chaudhari, and G. D. Barber, "Graphane: a two-dimensional hydrocarbon," Physical Review B-Condensed Matter and Materials Physics, vol. 75, no. 15, Article ID 153401, 2007.

[9] D. C. Elias, R. R. Nair, T. M. G. Mohiuddin et al., "Control of graphene's properties by reversible hydrogenation: evidence for graphane," Science, vol. 323, no. 5914, pp. 610-613, 2009.

[10] J. Zhou, Q. Wang, Q. Sun, X. S. Chen, Y. Kawazoe, and P. Jena, "Ferromagnetism in semihydrogenated graphene sheet," Nano Letters, vol. 9, no. 11, pp. 3867-3870, 2009.

[11] S. Y. Chou, M. S. Wei, P. R. Krauss, and P. B. Fischer, "Single-domain magnetic pillar array of $35 \mathrm{~nm}$ diameter and 65 Gbits/in. ${ }^{2}$ density for ultrahigh density quantum magnetic storage," Journal of Applied Physics, vol. 76, no. 10, pp. 66736675, 1994.

[12] D.-C. Li, L. Dai, S. Huang, A. W. H. Mau, and Z. L. Wang, "Structure and growth of aligned carbon nanotube films by pyrolysis," Chemical Physics Letters, vol. 316, no. 5-6, pp. 349$355,2000$. 
[13] S. C. Ray, C. W. Pao, H. M. Tsai et al., "A comparative study of the electronic structures of oxygen- and chlorinetreated nitrogenated carbon nanotubes by $\mathrm{x}$-ray absorption and scanning photoelectron microscopy," Applied Physics Letters, vol. 91, no. 20, Article ID 202102, pp. 1-3, 2007.

[14] S. Musso, S. Porro, M. Giorcelli, A. Chiodoni, C. Ricciardi, and A. Tagliaferro, "Macroscopic growth of carbon nanotube mats and their mechanical properties," Carbon, vol. 45, no. 5, pp. 1133-1136, 2007.

[15] M. S. Dresselhaus, G. Dresselhaus, R. Saito, and A. Jorio, "Raman spectroscopy of carbon nanotubes," Physics Reports, vol. 409, no. 2, pp. 47-99, 2005.

[16] A. C. Ferrari, J. C. Meyer, V. Scardaci et al., "Raman spectrum of graphene and graphene layers," Physical Review Letters, vol. 97, no. 18, Article ID 187401, 2006.

[17] A. C. Ferrari, "Raman spectroscopy of graphene and graphite: disorder, electron-phonon coupling, doping and nonadiabatic effects," Solid State Communications, vol. 143, no. 1-2, pp. 47-57, 2007.

[18] F. Tuinstra and J. L. Koenig, "Raman spectrum of graphite," Journal of Chemical Physics, vol. 53, no. 3, pp. 1126-1130, 1970.

[19] A. C. Ferrari and J. Robertson, "Interpretation of Raman spectra of disordered and amorphous carbon," Physical Review B, vol. 61, no. 20, pp. 14095-14107, 2000.

[20] W. Wu, Q. Yu, P. Peng, Z. Liu, J. Bao, and S.-S. Pei, "Control of thickness uniformity and grain size in graphene films for transparent conductive electrodes," Nanotechnology, vol. 23, no. 3, Article ID 035603, 2012.

[21] J. Stohr, NEXAFS Spectroscopy, Springer, Berlin, Germany, 1991.

[22] S. C. Ray, J. W. Chiou, W. F. Pong, and M.-H. Tsai, "The electronic properties of nanomaterials elucidated by synchrotron radiation-based spectroscopy," Critical Reviews in Solid State and Materials Sciences, vol. 31, no. 4, pp. 91-110, 2006.

[23] T. M. Whitney, J. S. Jiang, P. C. Searson, and C. L. Chien, "Fabrication and magnetic properties of arrays of metallic nanowires," Science, vol. 261, no. 5126, pp. 1316-1319, 1993.

[24] H. Ago, T. Kugler, F. Cacialli et al., "Work functions and surface functional groups of multiwall carbon nanotubes," Journal of Physical Chemistry B, vol. 103, no. 38, pp. 8116-8121, 1999.

[25] Z. Luo, J. Shang, S. Lim et al., "Modulating the electronic structures of graphene by controllable hydrogenation," Applied Physics Letters, vol. 97, no. 23, Article ID 233111, 2010.

[26] R. M. Bozorth, Ferromagnetism, D.Van Nostrand Company, New York, NY, USA, 1951.

[27] R. Kozhuharova, M. Ritschel, D. Elefant et al., "Well-aligned Cofilled carbon nanotubes: preparation and magnetic properties," Applied Surface Science, vol. 238, no. 1-4, pp. 355-359, 2004.

[28] C. T. Kuo, C. H. Lin, and A. Y. Lo, "Feasibility studies of magnetic particle-embedded carbon nanotubes for perpendicular recording media," Diamond and Related Materials, vol. 12, no. 3-7, pp. 799-805, 2003.

[29] F. Geng and H. Cong, "Fe-filled carbon nanotube array with high coercivity," Physica B: Condensed Matter, vol. 382, no. 1-2, pp. 300-304, 2006. 

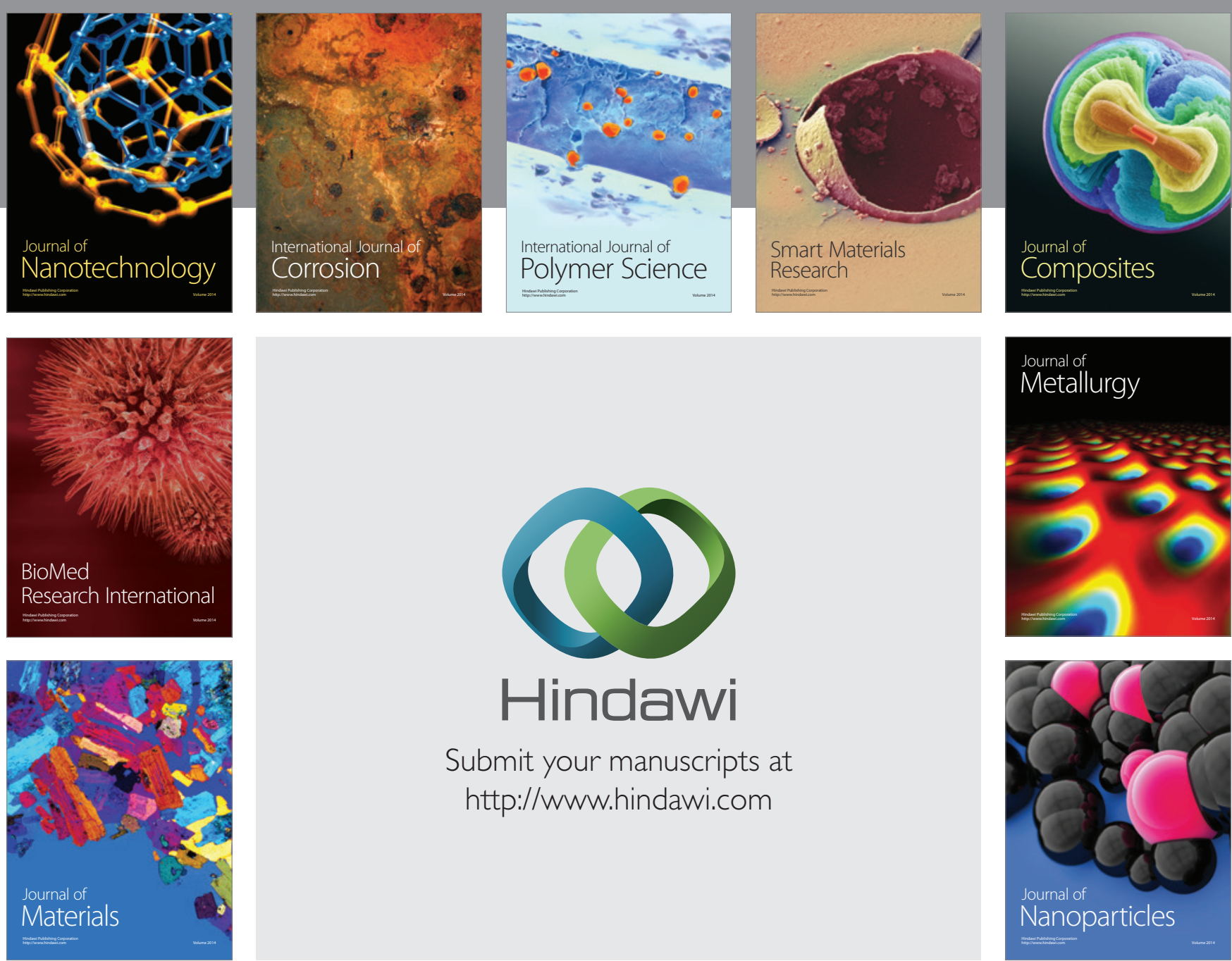

\section{Hindawi}

Submit your manuscripts at

http://www.hindawi.com

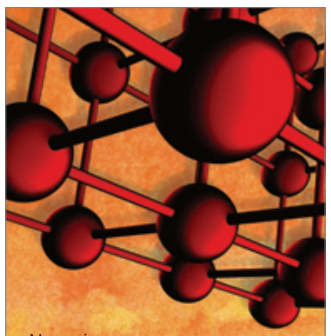

Materials Science and Engineering
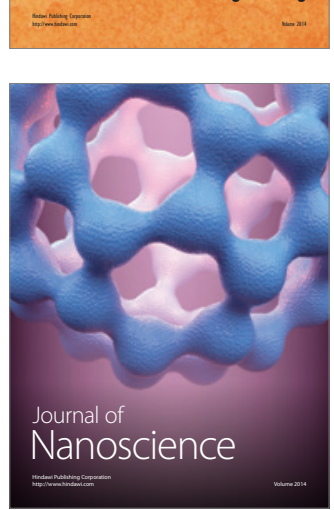
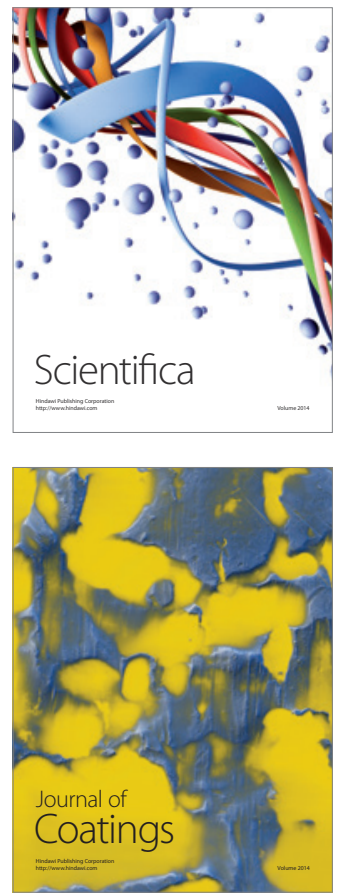
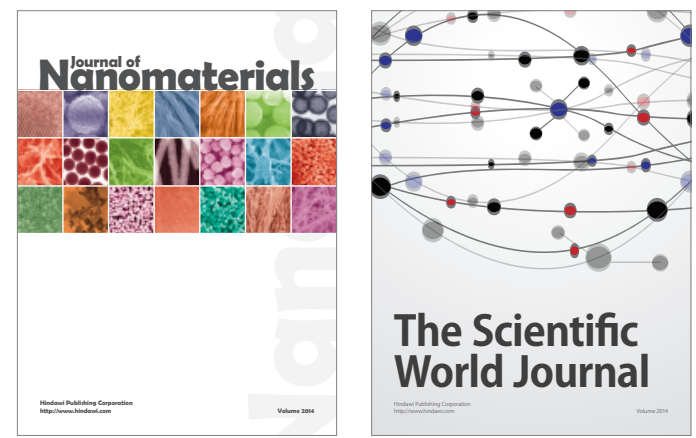

The Scientific World Journal
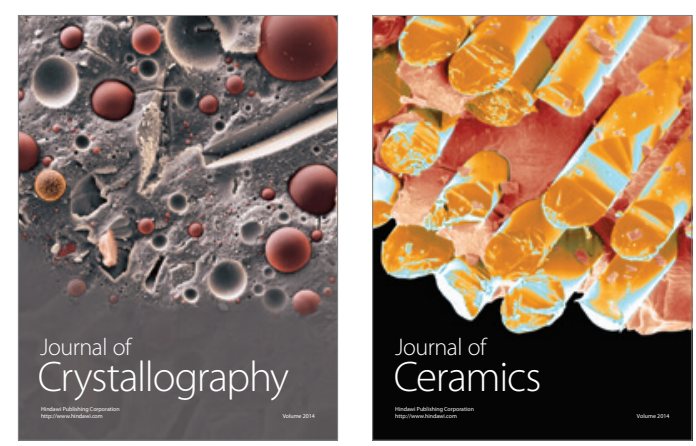
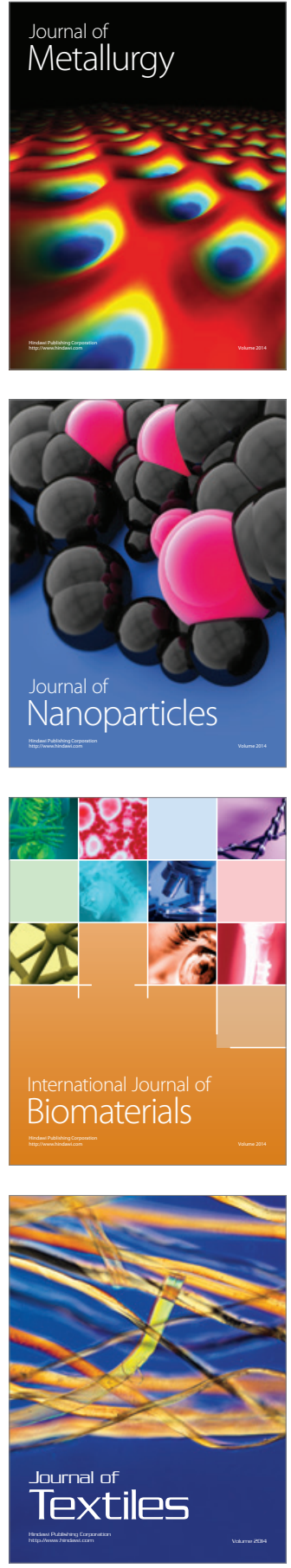